\title{
A 2D-Torus Based Packet Switching Optical Network-on-Chip Architecture
}

\author{
Lei Zhang, Emma E. Regentova, Xianfang Tan \\ Department of Electrical and Computer Engineering \\ University of Nevada, Las Vegas \\ zhang16@unlv.nevada.edu,regent@egr.unlv.edu, tanx@unlv.nevada.edu
}

\begin{abstract}
In this paper we present a new optical NoC architecture called GTON-XII. Data transmission in GTONXII is packet switching with different optical wavelengths through micro-ring resonators. Simulation results demonstrate GTON-XII is a high-bandwidth, low-cost, low-latency and lowpower-consuming architecture.
\end{abstract}

Keywords-optical NoC,packet switching, micro-ring
resonator

\section{INTRODUCTION}

In this paper, we propose a generalized 2D-torus-based Optical Network-on-Chip architecture called GTON-XII. The physical interconnection of GTON-XII is identical to a 2D-torus network, which is composed of waveguides and optical routers. Data transmission in GTON-XII is through direct optical channels. A direct optical channel (DOC) is a direct light path between two nodes composed of a set of interconnected physical links (waveguides) and optical interconnection units (OIU) based on micro-ring resonators (MRR). Each node in the GTON-XII has 12 DOCs connected to other different 12 nodes. By using a predetermined routing wavelength, light travels in a DOC without relay. As there existing more than one possible optical path between any two nodes, it is critical to choose the group of DOCs for a node so that minimal wavelengths needed for the 12 DOCs of that node. This paper presents a systematic method to select DOCs, assign wavelengths and design the optical routers. The simulation results show that GTON-XII is a high-bandwidth, low-cost, low-latency and low-power-consuming optical NoC architecture.

\section{GTON-XII ARCHITECTURE}

The switching element of GTON-II is shown in Fig.1, which is a MRR-based $2 \times 2$ optical switch. MRR is an optical device that can switch light between waveguides [1]. MRR resonantly tunnels light through it when the light frequency is within its pass band width and rejects it otherwise.

GTON-XII is an $N \times N$ (where $N=2 n, n \in \mathbf{N}$ ) 2D-torusbased network. Given a node (x,y) of GTON-XII, its channel map is shown in Fig.2. A $6 \times 6$ GTON-XII example is presented in Fig.3. Each node in the GTON-XII is presented by a pair $(\mathrm{x}, \mathrm{y})$ where $\mathrm{x}, \mathrm{y}=\{0,1, \ldots, N-1\}$. Each node has a router and a processor (core), and physically connects to 4 adjacent nodes.

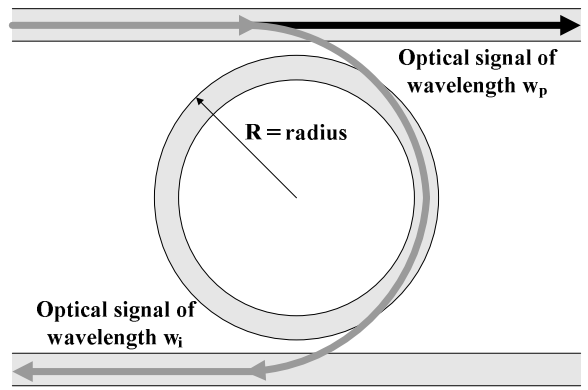

(a) Basic MRR structure

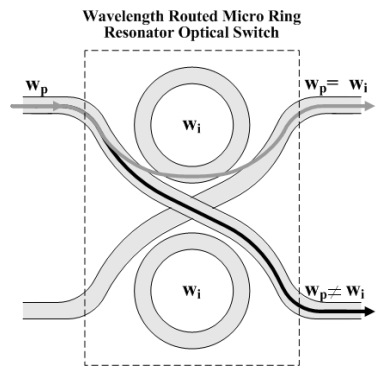

(b)

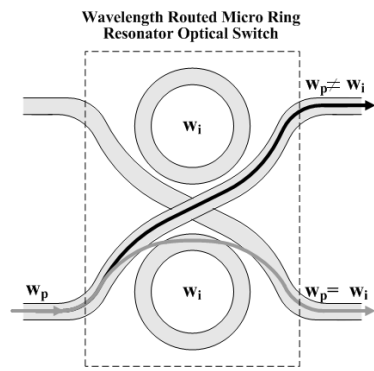

MRR-based switch
Figure 1. MMR-based optical switches.

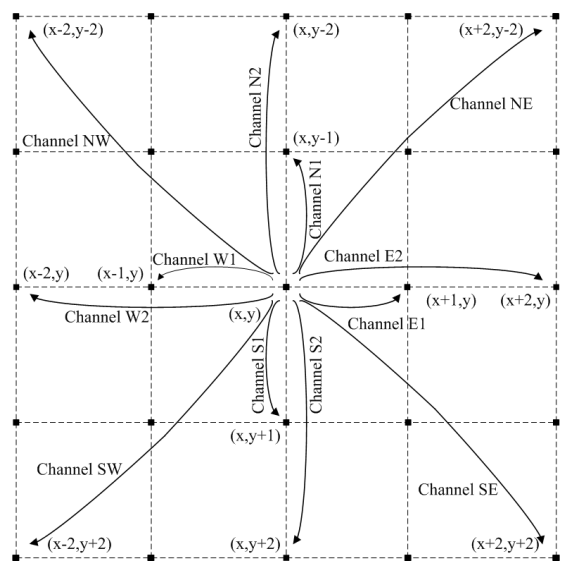

Figure 2. Node Channel map in GTON-XII

Optical router is the heart of an optical NOC. In GTONXII, each router internally has an OIU and an Optical 
Electrical Router (OER). OIU is a matrix switching device with six ports, among which four are external ports (S1, S2, $\mathrm{D} 1, \mathrm{D} 2)$, and two are internal ports (LS, LD) to connect to the OER.

The GTON-XII nodes are classified into two classes: INode associated with I-router; and II-node with II-router. Irouter has an I-OIU and an I-OER, and II-router has an IIIOU and an II-OER. Given coordinates (x,y), a node is an INode if $x+y=2 n(n \in \mathbf{N})$, otherwise it is a II-Node. From the interconnection of the $6 \times 6$ GTON-XII, it can be seen that for the I-Node at $(x, y)$, its $\mathrm{S} 1$ port is connected to the $\mathrm{S} 2$ port of the II-node at $(x, y-1), \mathrm{S} 2$ port is connected to the D2 of the II-node at $(x-1, y), \mathrm{D} 1$ is connected to the S1 of the II-node at $(x+1, y)$, and D2 is connected to the D1 of the II-node at $(x, y+1)$. And for the II-Node at $(x, y)$, its $\mathrm{S} 1$ port is connected to the D1 port of the I-node at $(x-1, y), \mathrm{S} 2$ is connected to the $\mathrm{S} 1$ of the I-node at $(x, y+1), \mathrm{D} 1$ is connected to the D2 of the I-node at $(x, y-1)$, and D2 is connected to the S2 of the I-node at $(x+1, y)$.

Data transmission in GTON-XII is via DOCs. Each node in the network is directly connected to 12 other nodes via 12 DOCs. The twelve DOCs are: $\mathrm{E} 1:(\mathrm{x}, \mathrm{y}) \leftrightarrow(\mathrm{x}+1, \mathrm{y}) ; \mathrm{W} 1$ : $(\mathrm{x}, \mathrm{y}) \leftrightarrow(\mathrm{x}-1, \mathrm{y}) ; \quad \mathrm{S} 1(\mathrm{x}, \mathrm{y}) \leftrightarrow(\mathrm{x}, \mathrm{y}+1) ; \mathrm{N} 1: \quad(\mathrm{x}, \mathrm{y}) \leftrightarrow(\mathrm{x}, \mathrm{y}-1) ; \quad \mathrm{E} 2:$ $(\mathrm{x}, \mathrm{y}) \leftrightarrow(\mathrm{x}+2, \mathrm{y}) ; \mathrm{W} 2:(\mathrm{x}, \mathrm{y}) \leftrightarrow(\mathrm{x}-2, \mathrm{y}), \mathrm{S} 2:(\mathrm{x}, \mathrm{y}) \leftrightarrow(\mathrm{x}, \mathrm{y}+2) ; \mathrm{N} 2:$ $(\mathrm{x}, \mathrm{y}) \leftrightarrow(\mathrm{x}, \mathrm{y}-2) ; \mathrm{SE}:(\mathrm{x}, \mathrm{y}) \leftrightarrow(\mathrm{x}+2, \mathrm{y}+2) ; \mathrm{NW}:(\mathrm{x}, \mathrm{y}) \leftrightarrow(\mathrm{x}-2, \mathrm{y}-2)$, NE: $(x, y) \leftrightarrow(x+2, y-2)$; and SW: $(x, y) \leftrightarrow(x-2, y+2)$. These DOC are grouped into three levels: Level 1 with channels $\mathrm{E} 1, \mathrm{~W} 1, \mathrm{~S} 1$ and N1, which are one-hop connections in $\mathrm{X}$ or $\mathrm{Y}$ direction; Level 2 with channels E2, W2, S2 and N2, which are two-hop connections in $\mathrm{X}$ or $\mathrm{Y}$ direction; and Level 3 with channels NE, NW, SE and SW, which are twohop diagonal connections. Examples of a one-hop DOC and a two-hop DOC in the $6 \times 6$ GTON-XII are shown in Fig.3.

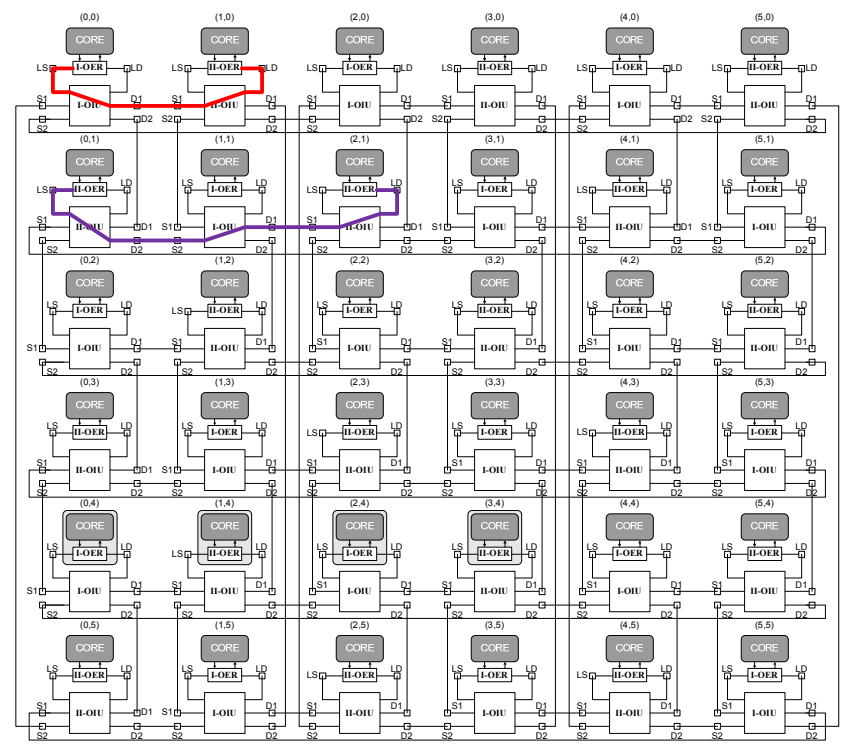

— : 1-hop DOC — : 2-hop DOC

Figure 3. A $6 \times 6$ GTON architecture and examples of DOCs.

\section{ROUTING DEVICES DESIGN IN GTON-XII}

OIU is a wavelength routed passive switching MRR matrix to route light between its 3 ports on each side according to the wavelength. The structure of OIU is constructed based on the concept of WRON [2]. The routing wavelengths assignment for all channels of I-Node and IINode is shown in Tab.1. I-OIU and II-OIU can be built with a series of optical switches as shown in Fig.4 to realize DOCs as shown in Fig.3.

\section{TABLE I. ROUTING TABLE FOR I-NODES AND II-NODES}

\begin{tabular}{|c|c|c|c|c|c|c|c|c|c|c|c|c|}
\hline \multicolumn{10}{|c|}{ I-Node } \\
\hline Channel & E1 & S1 & W1 & N1 & E2 & S2 & W2 & N2 & NE & SE & SW & NW \\
\hline Transmission Port & LS & LS & LD & LD & LS & LS & LD & LD & LS & LS & LD & LD \\
\hline Routing Wavelength & 8 & 9 & 8 & 9 & 7 & 10 & 7 & 10 & 6 & 4 & 6 & 2 \\
\hline \multicolumn{10}{|c|}{ II-Node } \\
\hline Channel & E1 & S1 & W1 1 & N1 & E2 2 & S2 & W2 & N2 & NE & SE & SW & NW \\
\hline Transmission Port & LS & LD & LD & LS & LS & LD & LD & LS & LS & LS & LD & LD \\
\hline Routing Wavelength & 8 & 9 & 8 & 9 & 10 & 7 & 10 & 7 & 1 & 5 & 3 & 5 \\
\hline
\end{tabular}

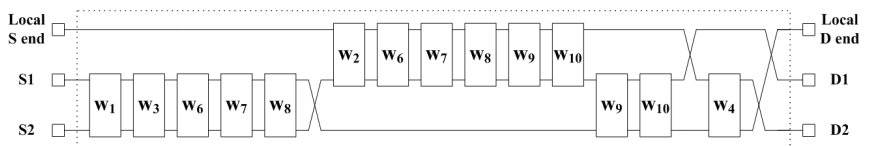

(a). I-OIU

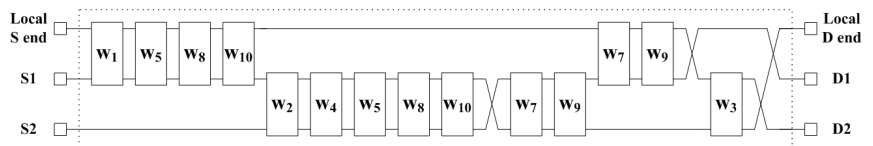

(b). II-OIU

Figure 4. I-OIU and II-OIU in GTON-XII

OER is to receive input optical signal and to convert it to electrical data, then read and analyze the data header to make routing decision. If relay is necessary, electrical to optical reconversion is performed with a proper wavelength to transmit to the next node. The OER in GTON-XII has 12 input and 12 output ports plus one input port and one output port to the local core. Demultiplexers on both ends of the OER separate multiple input optical signals and propagate each of them to the corresponding input port. A photodetector in each channel receives input optical signal and converts it to electrical data to the input buffer. In every OER, in each clock cycle, if an output channel is available, a frame will be switched to the output channel through the crossbar, and then it is converted to the optical signal and transmitted to next node. Frames destined to the local core will also be switched similarly yet no EO conversion is required. I-OER and II-OER can be built as shown in Fig.5. 


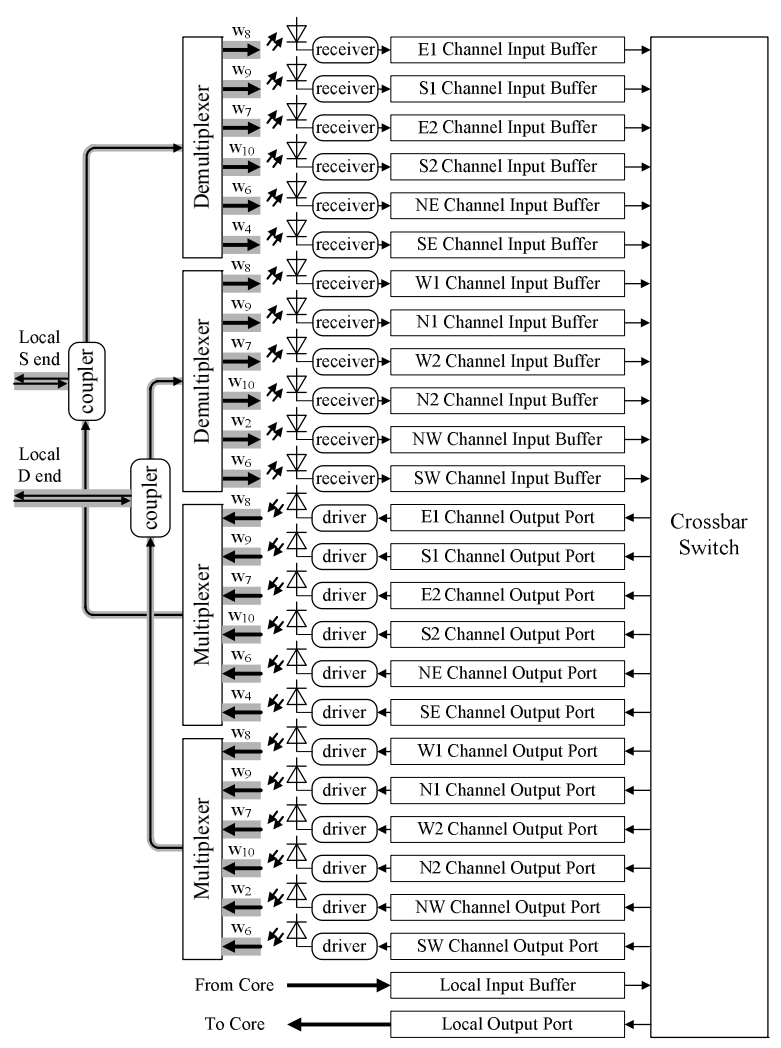

(a) I-OER in GTON-XII

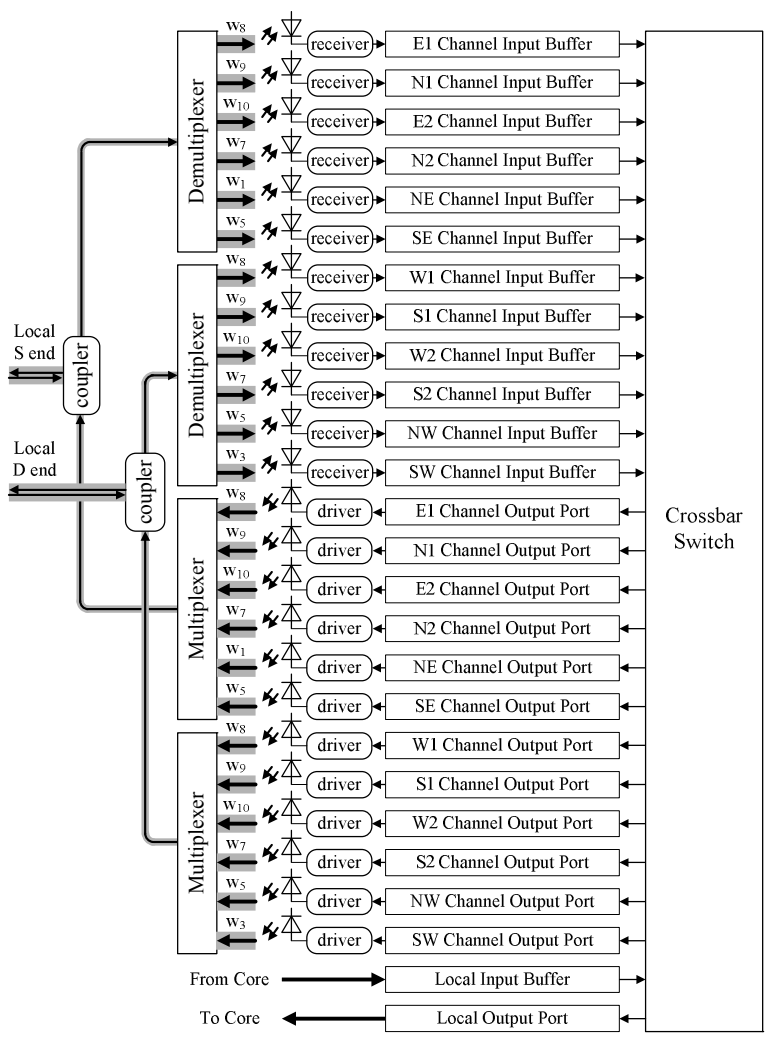

(b) II-OER in GTON-XII

Figure 5. I-OER and II-OER structures in GTON-XII

\section{NETWORK PROPERTIES OF GTON-XII}

Tab.2 compares the Diameter (D) and Average Distance (AD) of GTON-XII and a few other popular networks. 'Average Distance' denotes the number of hops, or the number of $\mathrm{OE}$ and $\mathrm{EO}$ conversions needed for data transmission between two nodes. Transmission power losses (dB) of all DOCs are listed in Tab.3 which are derived based on parameters according to [3]. And Tab.4 summarizes the architectural properties of the GTON-XII.

TABLE II. Diameter (D) AND AVERAGe Distance (AD) FOR DIFFERENT NETWORKS.

\begin{tabular}{|c|c|c|c|c|c|c|c|c|}
\hline \multirow{2}{*}{ Network } & \multicolumn{2}{|c|}{ GTON-XII } & \multicolumn{2}{c|}{ Mesh } & \multicolumn{2}{c|}{ 2D-Torus } & \multicolumn{2}{c|}{ Hypercube } \\
\cline { 2 - 9 } & $\boldsymbol{D}$ & $\boldsymbol{A D}$ & $\boldsymbol{D}$ & $\boldsymbol{A D}$ & $\boldsymbol{D}$ & $\boldsymbol{D S}$ & $\boldsymbol{D}$ & $\boldsymbol{A D}$ \\
\hline $8 \times 8$ & 3 & 2.13 & 14 & 5.33 & 8 & 4 & 6 & 3 \\
\hline $16 \times 16$ & 5 & 3.44 & 30 & 10.67 & 16 & 8 & 8 & 4 \\
\hline $32 \times 32$ & 9 & 6.09 & 62 & 21.33 & 32 & 16 & 10 & 5 \\
\hline
\end{tabular}

TABLE III. TAB. 3. TRANSMISSION POWER LOSS IN DOCS

\begin{tabular}{|c|c|c|c|c|c|c|c|c|c|c|c|c|c|c|}
\hline D) & & $E$ & & $W 1$ & $N 1$ & $E 2$ & $\overline{S 2}$ & $W^{2}$ & $N 2$ & $N E$ & $\overline{S E}$ & $\overline{S W}$ & & Avg. \\
\hline \multirow{2}{*}{ I-Node } & $\mathrm{W}$. & 8 & 9 & 8 & 9 & 7 & 10 & 7 & 10 & 6 & 4 & 6 & 2 & \\
\hline & Loss & 5.72 & 7.15 & 5.9 & 3.6 & 7.7 & 7.8 & 47.72 & 5.06 & 9.35 & 7.81 & 9.35 & 7.81 & \\
\hline & W. & \begin{tabular}{|l|}
8 \\
\end{tabular} & 9 & 8 & 9 & 10 & 7 & 10 & 7 & 1 & 5 & 3 & 5 & 0 \\
\hline
\end{tabular}

TABLE IV. ARCHITECTURE PROPERTIES OF GTON-XII

\begin{tabular}{|c|c|}
\hline Architecture Properties & Value \\
\hline Channels of each node & 12 \\
\hline Lasers in each node & 12 \\
\hline Photodetectors in each node & 12 \\
\hline Routing wavelengths in each node & 7 \\
\hline Total routing wavelengths in the network & 10 \\
\hline MRRs in each node & 28 \\
\hline MRR optical switches in each node & 14 \\
\hline Electrical input queues in each node & 12 \\
\hline Electrical output queues in each node & 0 \\
\hline Optical buffers in each node & 0 \\
\hline Optical couplers in each node & 2 \\
\hline Electrical crossbar switches in each node & 1 \\
\hline Optical wavelength multiplexers in each node & 2 \\
\hline Optical wavelength demultiplexers in each node & 2 \\
\hline TO/EO tuning devices in the network & 0 \\
\hline
\end{tabular}

\section{Simulation}

We test the maximum traffic load the network can handle without blocking. The network is set to be homogeneous; and all nodes have the same frame generating rate. The parameter setting of the simulation is shown in the Tab.5.

TABLE V. Simulation SetTing

\begin{tabular}{|c|c|}
\hline Network Pattern & Homogeneous \\
\hline Optical Frequency & $40 \mathrm{G}$ \\
\hline Electrical Frequency & $4 \mathrm{G}$ \\
\hline Packet Length & 256 bits \\
\hline Electrical Bus Width & 64 \\
\hline Packet Generating Rate & $2.4 \times 10^{-3} \sim 0.08$ packet/nanosecond \\
\hline Packet Generation Pattern & Uniform Distribution \\
\hline Network Size & $6 \times 6 \sim 16 \times 16$ \\
\hline Routing Algorithm & Deterministic Routing \\
\hline Buffer service & FIFO \\
\hline
\end{tabular}


In the simulation, for each setting at least 10,000 frames are generated by each node and transmitted to all other nodes with the same probability. The total time transmission of per is measured from the generation till the receival by its destination core. The deterministic routing algorithm is applied, in which data are transmitted according to the following sequence: Level3 channels first, Level2 channels second and Level1 channels last. In Level1 channels routing the sequence of $\mathrm{x}-\mathrm{y}$ is applied to minimize deadlock.

The average frame transmission time in GTON-XII of different sizes and with different frame generating rates are shown in Fig.6. It can be observed that before a point when networks reaches the blocking condition the waiting time is negligible.

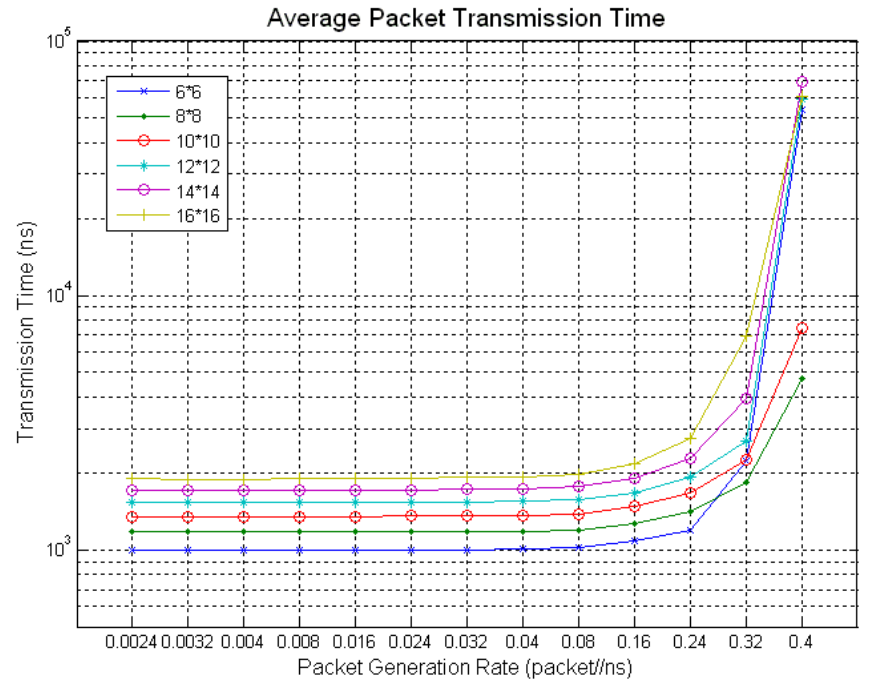

Figure 6. Average packet transmission time in GTON-XII

\section{CONCLUSION}

In this paper, we proposed a generalized 2D-torus based packet switching optical NoC architecture with the use of micro-ring resonator (MRR) optical switches. GTON-XII is highly scalable because of packet switching used. The use of passive MRR switching, WDM [4] and DOC offers advantages such as extremely high bandwidth, low latency, low cost, low power consumption, and excellent fault tolerance. The performance of the GTON-XII is evaluated by simulation. In conclusion, the GTON has shown many outstanding features which make it a compelling solution for future optical NoCs.

\section{REFERENCES}

[1] J. Xue, A. Garg, B. Ciftcioglu, S. Wang, I. Savidis, J. Hu, M. Jain, M. Huang, H. Wu, E. G. Friedman, G. W. Wicks and D. Moore, "An Intra-Chip Free-Space OpticalInterconnect", Proc. 37thAnnual Int'l Symp. on Computer Architecture, pp. 94-105, 2010

[2] L. Zhang, M. Yang, Y. Jiang and E. E. Regentova, "Architectures and Routing Schemes for Optical Network-on-Chips", Computers \& Electrical Engineering, vol. 35, Issue 6, pp. 856-877, Nov. 2009.

[3] C. Batten, A. Joshi, J. Orcutt, A. Khilo, B. Moss, C. Holzwarth, M. Popovic, H. Li, H. Smith, J. Hoyt, F. Kärtner, R. Ram, V. Stojanovic and K. Asanovic, "Building Manycore Processor-to-DRAM Networks with Monolithic Silicon Photonics", Proc. IEEE Symp. on HighPerformance Interconnects, pp. 21-30, Aug. 2008.
[4] S. Xiao, M. H. Khan, H. Shen and M. Qi, "Multiple-channel silicon micro-resonator based filters for WDM applications", Opt. Express, vol. 15 , issue 12 , pp. 7489-7498, 2007 